\title{
Ultra-thin 3D silicon sensors for neutron detection
}

\section{Guardiola, Consuelo}

2012-03-15

Guardiola , C , Fleta , C , Pellegrini , G, Garcia , F, Quirion, D , Rodriguez , J \& Lozano , M 2012 , ' Ultra-thin 3D silicon sensors for neutron detection ' , Journal of Instrumentation, vol. 7 , pp. P03006 . https://doi.org/10.1088/1748-0221/7/03/P03006

http://hdl.handle.net/10138/32409

https://doi.org/10.1088/1748-0221/7/03/P03006

publishedVersion

Downloaded from Helda, University of Helsinki institutional repository.

This is an electronic reprint of the original article.

This reprint may differ from the original in pagination and typographic detail.

Please cite the original version. 
Ultra-thin 3D silicon sensors for neutron detection

This article has been downloaded from IOPscience. Please scroll down to see the full text article.

2012 JINST 7 P03006

(http://iopscience.iop.org/1748-0221/7/03/P03006)

View the table of contents for this issue, or go to the journal homepage for more

Download details:

IP Address: 128.214.56.126

The article was downloaded on 15/03/2012 at 11:37

Please note that terms and conditions apply. 


\title{
Ultra-thin 3D silicon sensors for neutron detection
}

\author{
C. Guardiola,,${ }^{a} 1$ C. Fleta, ${ }^{a}$ G. Pellegrini, ${ }^{a}$ F. García, ${ }^{b}$ D. Quirion, ${ }^{a}$ J. Rodríguez ${ }^{a}$ and \\ M. Lozano ${ }^{a}$
}

\author{
${ }^{a}$ Instituto de Microelectrónica de Barcelona, (IMB-CNM, CSIC), \\ 08193 Bellaterra (Barcelona), Spain \\ ${ }^{b}$ Helsinki Institute of Physics, University of Helsinki, \\ 00014 Helsinki, Finland \\ E-mail: Consuelo.Guardiola@imb-cnm.csic.es
}

ABSTRACT: We present a novel neutron detector based on an ultra-thin 3D silicon sensor with a sensitive volume only $10 \mu \mathrm{m}$ thick. This ultra-thin active volume allows a high gamma-ray rejection, a key requirement in order to discriminate the signal coming from the neutrons in a mixed neutron-gamma ray environment. The device upper-side is covered with a novel boronbased compound that detects neutrons by means of the ${ }^{10} \mathrm{~B}(\mathrm{n}, \alpha){ }^{7} \mathrm{Li}$ nuclear reaction.

The performance of test devices has been investigated first with a gamma-ray source to evaluate the gamma-ray rejection factor, and then with an ${ }^{241} \mathrm{AmBe}$ neutron source to assess the neutrongamma ray discrimination properties.

KEYWORDS: Solid state detectors; Neutron detectors (cold, thermal, fast neutrons)

\footnotetext{
${ }^{1}$ Corresponding author. Consuelo Guardiola, Radiation Detectors Group, Instituto de Microelectrónica de Barcelona IMB-CNM (CSIC).
} 


\section{Contents}

1 Introduction 1

2 Adapted ultra-thin 3D detectors 3

2.1 U3DTHIN 3

2.2 U3DTHIN adapted for neutron detection 5

3 Measurements $\quad 7$

3.1 Gamma rejection factor

3.2 Gamma-Neutron discrimination 7

4 Conclusions 9

\section{Introduction}

In many environments where the neutron field is a concern, such as space exploration, nuclear security or medical radiotherapy rooms, neutrons are mixed with a large gamma ray background. In order to detect these neutrons, it is necessary to discriminate them from the gamma rays. This is an issue that relies strongly on the gamma ray spectrum, and therefore, on the kind of the detector used. Thus, two factors are fundamental to evaluate whether a detector is useful in a mixed neutrongamma ray environment: the neutron-gamma discrimination rate and the gamma rejection.

There are three main types of detectors based on the ionization produced by charged particles in matter [1]: ionization of gases, ionization in semiconductors, and scintillators. All these detection methods are based on the fact that charged particles or photons ionize the sensitive volume of the detector, inducing a small electrical pulse. In contrast, neutrons are not charged particles so they are not able to cause direct ionization processes by Coulombian interaction. However neutrons may produce charged particles in some materials by direct scattering of the nuclei or as nuclear reaction products, the cross-section of each process depending on the material. The charged particles product of the neutron reaction can be subsequently detected by an ionization-based detector. Fast neutrons are usually detected via the protons produced by elastic scattering with the hydrogen nuclei of the neutron moderator material (water, polyethylene or paraffin). For slow neutrons, an isotope which captures neutrons to produce charged particles is normally used. The most common converter materials for slow neutrons are listed in table 1.

${ }^{3} \mathrm{He}$ tube-based detectors have been the preferred solution to obtain high gamma-neutron discrimination for decades [2]. These detectors work in the pulse-readout mode where neutron and gamma-ray signals are distinguished by their amplitude. However, such discrimination is hindered by collisions of the reaction products with the tube walls, resulting in an additional pulse height spectrum. 
Table 1. Converters to capture thermal neutrons.

\begin{tabular}{|c|c|c|}
\hline Isotope & Neutron Capture Reaction & $\sigma_{t h}$ (barn) \\
\hline${ }^{10} \mathrm{~B}$ & ${ }^{10} \mathrm{~B}+n \stackrel{94 \%}{\rightarrow} \alpha(1.47 \mathrm{MeV})+{ }^{7} \mathrm{Li}(0.84 \mathrm{MeV})+\gamma(0.48 \mathrm{MeV})$ & 3840 \\
& $\stackrel{6 \%}{\rightarrow} \alpha(1.78 \mathrm{MeV})+{ }^{7} \mathrm{Li}(1.01 \mathrm{MeV})$ & \\
\hline${ }^{6} \mathrm{Li}$ & $n+{ }^{6} \mathrm{Li} \rightarrow{ }^{3} \mathrm{H}(2.72 \mathrm{MeV})+\alpha(2.05 \mathrm{MeV})$ & 940 \\
\hline${ }^{3} \mathrm{He}$ & $n+{ }^{3} \mathrm{He} \rightarrow{ }^{3} \mathrm{H}(191 \mathrm{keV})+p(573 \mathrm{keV})$ & 5330 \\
\hline
\end{tabular}

The ${ }^{6} \mathrm{Li}$ is normally used in neutron-sensitive inorganic scintillators through LiI doped with $\mathrm{Eu}$. The highly enriched ${ }^{6} \mathrm{LiI}(\mathrm{Eu})$ crystals $\left(96 \%{ }^{6} \mathrm{Li}\right)$ exhibit high thermal neutron detection efficiency [3].

However both ${ }^{3} \mathrm{He}$ and ${ }^{6} \mathrm{Li}$-based scintillator detectors share some undesirable characteristics: not only they are bulky and require costly operating conditions (high pressures ( $\sim 10$ bar) and voltages $(\sim 10 \mathrm{kV})$ for ${ }^{3} \mathrm{He}$ detectors; connection to bulky photomultiplier working at hundred of volts, need for hermetic assembly due to the hygroscopic nature of $\mathrm{LiI}(\mathrm{Eu})$ crystals for ${ }^{6} \mathrm{Li}$-based scintillators), exhibit slow-rise time, but also face difficulties in material supply: ${ }^{3} \mathrm{He}$ and ${ }^{6} \mathrm{Li}$ are expensive, ${ }^{3} \mathrm{He}$ reserves are foreseen to be consumed in the coming decades [4] and ${ }^{6} \mathrm{Li}$ requires special authorizations for exportation. These features do not allow for the versatility that some environments require and jeopardize the full applicability of these detectors.

In contrast, semiconductor-based detectors offer many useful features, such as compactness and robustness, low weight, bias voltage, and battery consumption and a high count rate capability [5]. These advantageous characteristics make them ideal for portable systems which require integrated signal acquisition and low power. Their compactness originates from the high density of the semiconductor material which allows a short range of the nuclear reaction products, but also increases the probability of interaction of the gamma-rays. The ideal semiconductor candidate for neutron detection is silicon, which provides a relatively low $\mathrm{Z}$ for moderate gamma-ray interaction probability and above all a high technological know-how thanks to the microelectronics industry which makes it a material of choice as active sensor.

To ensure a good neutron-gamma ray discrimination, a compromise in the active thickness of the sensor has to be found. Ideally the active thickness should match exactly the range of the reaction products in the material, on the order of few microns in silicon for the reactions listed in table I. However, in the usual parallel plate geometry for silicon detectors, such a low thickness comes usually at the expense of a high capacitance and therefore large electronic noise. In contrast, we present an ultra-thin 3D silicon sensor of $10 \mu \mathrm{m}$ thick with low capacitance, and therefore with low electronic noise, which leads to detect neutrons.

As explained above, in order to adapt a silicon detector for neutron detection, a suitable neutron converter must be used, preferably a converter whose capture products emit charged particles that are able to reach the sensitive volume of the detector. This converter should have a high neutron absorption cross section over a wide neutron energy range and a quantum yield nominally approximate to 1 . In this work we focus on ${ }^{10} \mathrm{~B}$ as neutron converter. When a ${ }^{10} \mathrm{~B}$ nucleus captures a neutron, it produces two charged particles, a ${ }^{7} \mathrm{Li}$ nucleus and an alpha particle (see table 1) that 


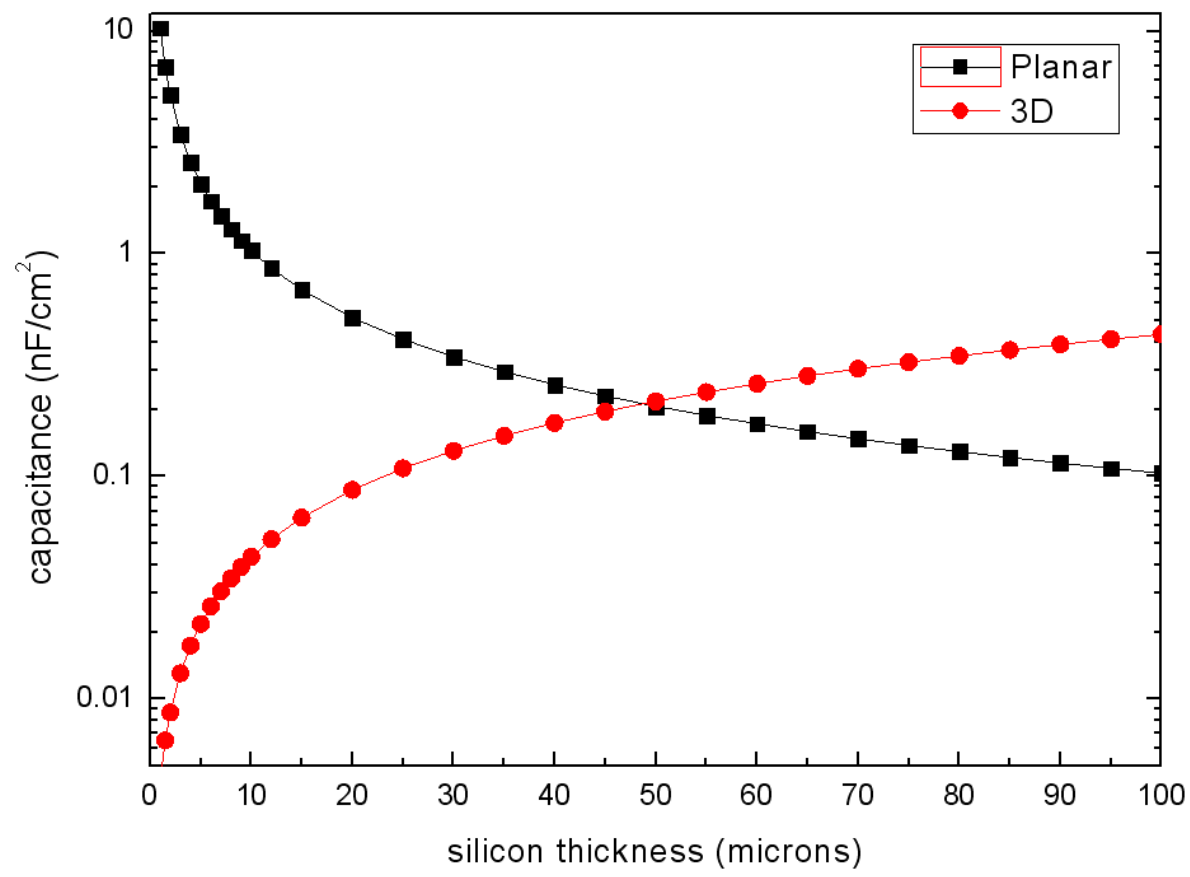

Figure 1. Comparison between the detector capacitance as a function of the silicon thickness for planar and U3DTHIN silicon detectors with $80 \mu \mathrm{m}$ pitch. The parallel-plate and cylindrical capacitor approximations, respectively, were used. The U3DTHIN structure is advantageous for thicknesses lower than $50 \mu \mathrm{m}$.

will be detected by the detector if they reach the silicon sensitive-volume with enough energy and create a signal over the minimum threshold.

\section{Adapted ultra-thin 3D detectors}

\subsection{U3DTHIN}

The neutron detectors used in this work are adapted ultra-thin 3D silicon sensors, U3DTHIN [6][8]. The U3DTHIN is fabricated on a Silicon-on-Insulator (SOI) wafer to reduce the thickness to only tens of microns. The use of silicon sensors with thin active layers for neutron detection applications is already well known [9]-[11] but all these devices are based on a planar geometry (i.e., they deplete vertically from the surface) and thus suffer from a high junction capacitance. In contrast, the U3DTHIN is based on the 3D technology [12]. In the U3DTHIN the collecting electrodes are columns etched through the silicon instead of being deposited on the surface like in the standard planar diodes. The lateral depletion of these devices allows for a much lower capacitance (hence a lower electronic noise) compared to a planar sensor of the same thickness. This can be seen in figure 1 that compares the capacitance of a standard silicon detector with that of the 3D structure. For a silicon thickness of $10 \mu \mathrm{m}$, the U3DTHIN capacitance is two orders of magnitude smaller than a planar silicon detector with the same thickness and surface area.

The U3DTHIN sensors used for these tests were designed and produced at the facilities of the Instituto de Microelectrónica de Barcelona (IMB-CNM) clean room [13]. The active thickness of 


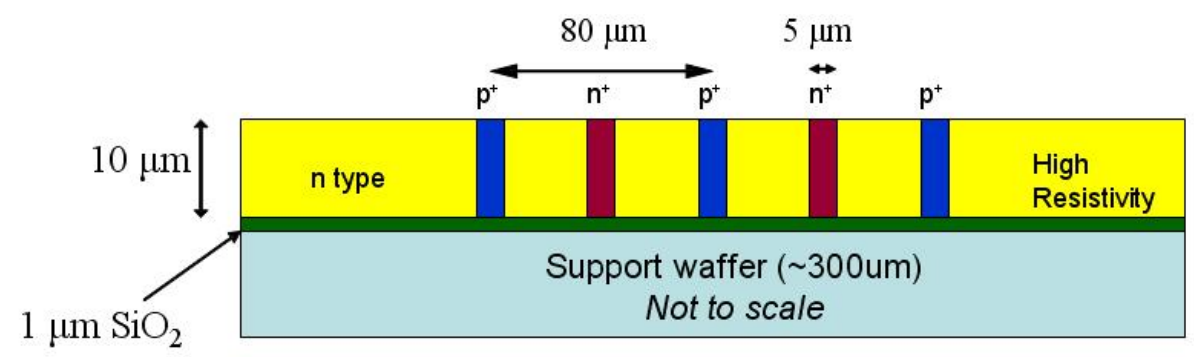

Figure 2. Lateral layout of the U3DTHIN detector. The converter layer is not shown in the sketch.

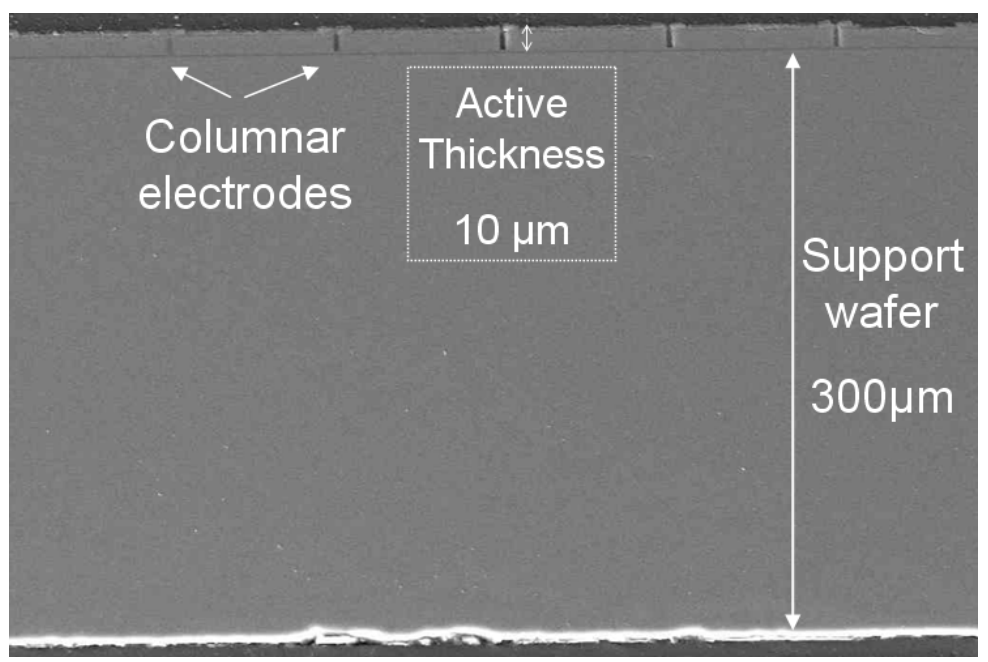

Figure 3. SEM image of the cross-section of the U3DTHIN structure.

the devices is only $10 \mu \mathrm{m}$. Figures 2 and 3 show a schematic view of the structure and an image of the section of the fabricated devices.

The columnar electrodes are distributed in a square geometry with $80 \mu \mathrm{m}$ pitch between the same type columns, with a total area of $1 \mathrm{~cm}^{2}$. The wafers used for the fabrication were SOI wafers with a high resistivity n-type active silicon layer $10 \mu \mathrm{m}$ thick. The silicon etched for the electrodes was done with an Alcatel 601E machine to produce holes with $5 \mu \mathrm{m}$ diameter and $10 \mu \mathrm{m}$ depth. Next, the columns were partially filled with polysilicon and doped with boron $\left(\mathrm{p}^{+}\right)$or phosphorous $\left(\mathrm{n}^{+}\right)$to form the $\mathrm{p}-\mathrm{n}$ junction or the ohmic contact, respectively, and were passivated with a thin oxide layer. Details of the fabrication technology can be found in [7]. Finally, the electrodes of the same type were connected together with an Al layer to provide the electrical contact. The full depletion voltage of the fabricated devices is $5 \mathrm{~V}$, the breakdown voltage is higher than $150 \mathrm{~V}$ and the device leakage current at full depletion is $3 \mathrm{nA} / \mathrm{cm}^{2}$.

Figure 4 shows an U3DTHIN device connected to the custom-built readout electronics used to carry out the tests. The electronics is described in [14].

The detectors were calibrated using an alpha-emitting ${ }^{241} \mathrm{Am}$ source. This isotope emits alpha particles of $5.5 \mathrm{MeV}(85 \%)$ and $5.443 \mathrm{MeV}(13 \%)$. The measurement was carried out in air, with a collimator and at $2 \mathrm{~cm}$ distance from the detector. Figure 5 shows the pulse distribution of the signal of the U3DTHIN with this source. 


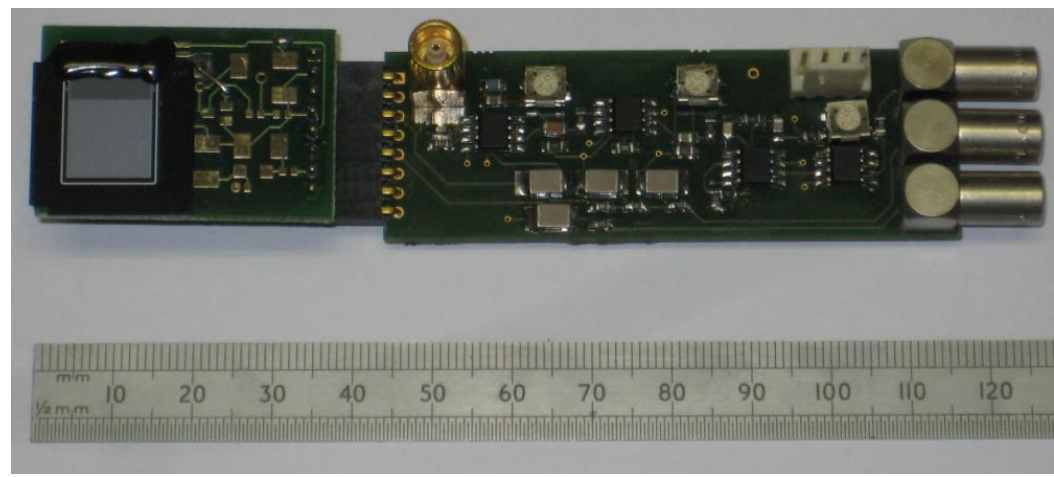

Figure 4. Picture of the U3DTHIN connected to the readout electronics.

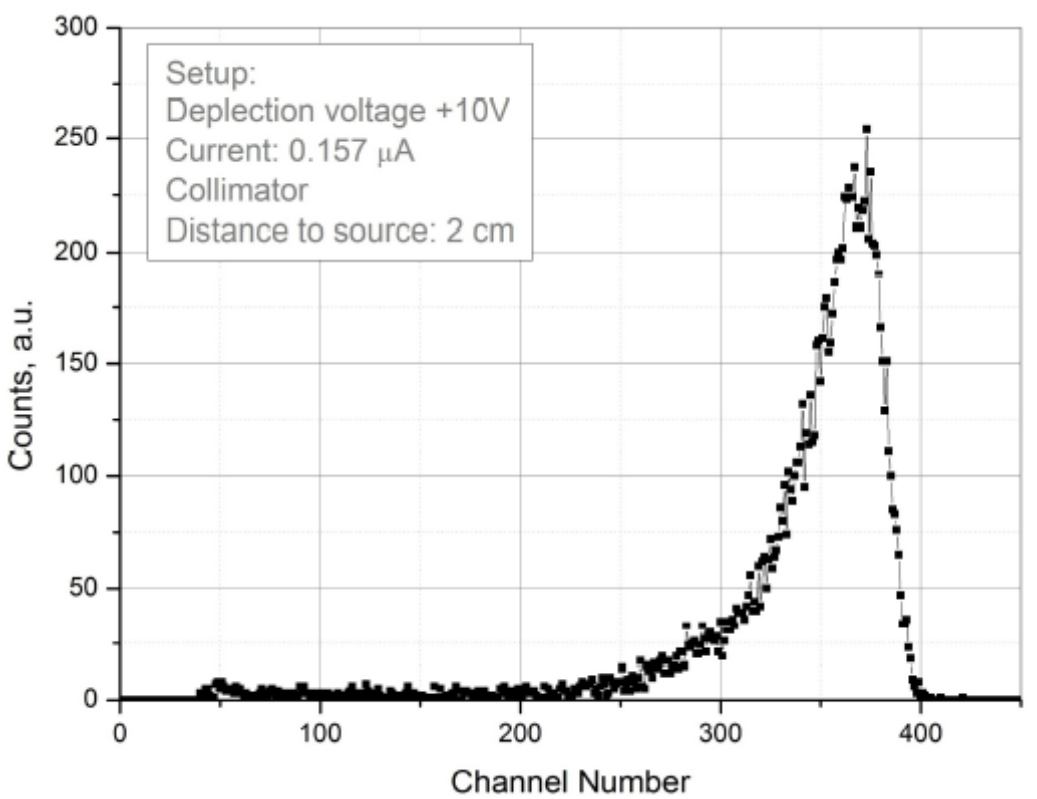

Figure 5. Signal distribution of the U3DTHIN produced by a ${ }^{241} \mathrm{Am}$ alpha source.

\subsection{U3DTHIN adapted for neutron detection}

In order to adapt the U3DTHIN, a boron-based converter layer was deposited on its front surface. The converter used is an o-carborane $\left(\mathrm{C}_{2} \mathrm{~B}_{10} \mathrm{H}_{12}\right)$ [15], dissolved in a cyanoacrylate matrix within tetrahydrofuran (THF). After this solution is deposited, the THF evaporates, leaving an adhesive matrix of o-carborane/cyanoacrylate with a density of $1.37 \mathrm{~g} / \mathrm{cm}^{3}$. The composition of this compound is shown in the table 2 . This material was chosen for the converter layer as it is easy to deposit on devices or even full wafers by spinning, thus allowing for an easy industralization of the fabrication process.

The thickness of the deposited converter layer was previously optimized with MCNPX simulations [16]. Figure 6 shows a schematic view of the simulated model, which includes: (i) the geometric dimensions and the components of the detector as well as the converter thickness to optimize, (ii) physics models for the neutron tracking, and (iii) the interaction of a thermal neutrons 
Table 2. Converter composition.

\begin{tabular}{|c|c|}
\hline Materials & Mass Fraction [\%] \\
\hline \multirow{2}{*}{$o$-carborane and cyanoacrylate } & $81.9 \% o$-carborane \\
\cline { 2 - 2 } & $18.11 \%$ cyanoac. \\
\hline $\mathrm{H}$ & 7.5 \\
\hline $\mathrm{C}$ & 28.7 \\
\hline${ }^{10} \mathrm{~B}$ & 53.0 \\
\hline $\mathrm{O}$ & 7.5 \\
\hline $\mathrm{N}$ & 3.3 \\
\hline
\end{tabular}

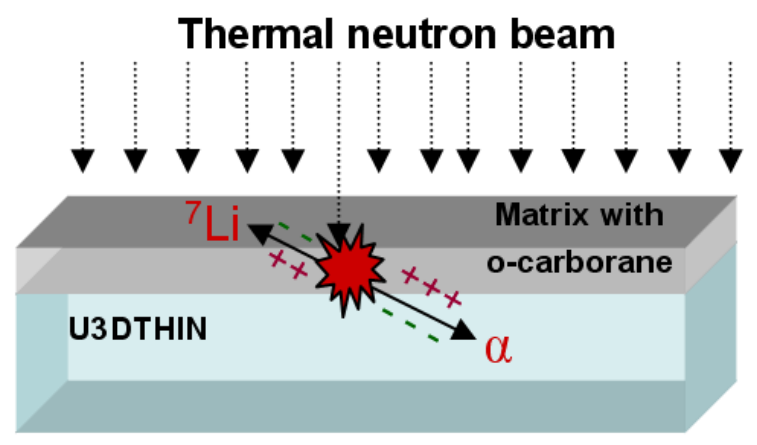

Figure 6. Schematic view of the simulated geometry of the adapted U3DTHIN in the MCNPX model.

beam focused towards the entrance face of the detector. To estimate the optimum converter thickness, the detection efficiency was calculated. This is defined as the ratio of neutrons detected to the total neutrons entering the device.

Figure 7 shows the thermal neutron efficiencies of the simulated detectors predicted with MCNPX for the $\mathrm{C}_{2} \mathrm{~B}_{10} \mathrm{H}_{12} /$ cyanoacrylate mixture. It can be seen that the efficiency for thermal neutrons can be up to $2.5 \%$ for a $10 \mu \mathrm{m}$ layer of the $\mathrm{C}_{2} \mathrm{~B}_{10} \mathrm{H}_{12} /$ cyanoacrylate compound fabricated with ${ }^{10} \mathrm{~B}$ or $0.5 \%$ for the same layer with natural $\mathrm{B}\left(20 \%{ }^{10} \mathrm{~B}\right)$. Thus, adhesive films of the ocarborane compound with ${ }^{10} \mathrm{~B}$ with an average thickness of $10 \mu \mathrm{m}$ were deposited onto the surface of the U3DTHIN devices.

Further simulations carried out with Geant $4[6]$ assure that the ${ }^{10} \mathrm{~B}(\mathrm{n}, \alpha){ }^{7} \mathrm{Li}$ reaction products, with the energies listed in table 1 , deposit all their energy into the $10 \mu \mathrm{m}$ sensitive bulk. This is due to the fact that the mean ranges of these reaction products within silicon are lower than $10 \mu \mathrm{m}$ : $5.15 \mu \mathrm{m}$ and $6.36 \mu \mathrm{m}$ for alphas with $1.47 \mathrm{MeV}$ and $1.78 \mathrm{MeV}$, and $2.46 \mu \mathrm{m}$ and $2.80 \mu \mathrm{m}$ for ${ }^{7} \mathrm{Li}$ ions with $0.84 \mathrm{MeV}$ and $1.01 \mathrm{MeV}$, energies respectively (these values are estimations by the SRIM software [17]). This means that the U3DTHIN thickness allows a dual function: it is sufficiently thin to capture the reaction products coming from the ${ }^{10} \mathrm{~B}(\mathrm{n}, \alpha)^{7} \mathrm{Li}$ and at the same time to reject a wide gamma spectrum. 


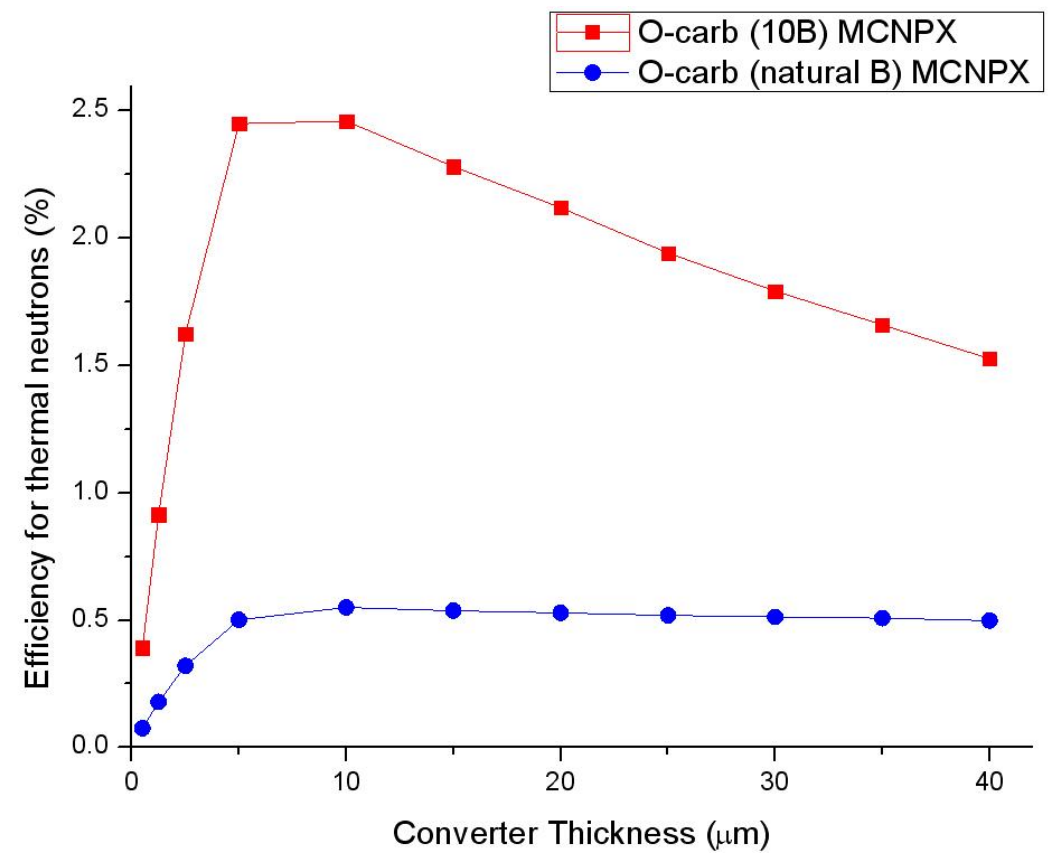

Figure 7. Simulated thermal neutron detection efficiency for an adapted U3DTHIN detector using the o-carborane/cyanoacrylate compound with ${ }^{10} \mathrm{~B}$ and natural boron, as a function of the converter thickness.

Table 3. ${ }^{137}$ Cs source characteristics.

\begin{tabular}{|c|c|c|c|}
\hline Nuclide & Half-life (y) & Energy $\boldsymbol{\gamma}$-ray [keV] & Activity [Bq] \\
\hline${ }^{137} \mathrm{Cs}$ & 30.17 & 661.657 & $3.310^{9}$ \\
\hline
\end{tabular}

\section{Measurements}

\subsection{Gamma rejection factor}

A ${ }^{137} \mathrm{Cs}$ source was used to assess the gamma rejection factor of the U3DTHIN. Table 3 shows the characteristics of the source.

The U3DTHIN was located $1 \mathrm{~m}$ distance from the ${ }^{137} \mathrm{Cs}$ source which emitted isotropically. An energy threshold of $100 \mathrm{keV}$ was applied to the readout system to avoid electronic noise. In these conditions, only one count was measured for the $10 \mu \mathrm{m}$ thickness detector for a measuring time of 10 minutes. This means that the gamma rejection factor of the U3DTHIN for the $662 \mathrm{keV}$ photons and an energy threshold of $100 \mathrm{keV}$ is higher than $10^{-8}$. Under the same experimental conditions, standard silicon detectors with thicknesses $300 \mu \mathrm{m}$ and $800 \mu \mathrm{m}$ registered total count rates of $(29.0 \pm 0.2)$ counts/s and (204.0 \pm 0.6$)$ counts/s respectively.

\subsection{Gamma-Neutron discrimination}

The U3DTHIN devices were exposed to an ${ }^{241} \mathrm{AmBe}$ neutron source with an activity of $40 \mathrm{mCi}$, and a total neutron emission rate of $8.8 \cdot 10^{4} \mathrm{n} / \mathrm{s} \cdot 4 \pi$ sr (i.e. 2200 neutrons/s per mCi of ${ }^{241} \mathrm{Am}$, with neutron average energy of approximately $4.5 \mathrm{MeV})$. The ${ }^{241} \mathrm{AmBe}$ source also produces photons with 
Table 4. Gamma and neutron emision per $10^{6}$ alphas of ${ }^{241} \mathrm{Am}-\mathrm{Be}$ source.

\begin{tabular}{|c|c|c|c|}
\hline \multirow{2}{*}{ Particle emitted } & \multirow{2}{*}{ Emitted from } & \multirow{2}{*}{ Energy } & $\begin{array}{c}\text { Intensity } \\
\text { (particles } / \mathbf{1 0}^{6} \text { alphas) }\end{array}$ \\
\hline & & & $\begin{array}{c}{ }^{241} \mathrm{AmBe} \\
{ }_{40} \mathrm{mCi}\end{array}$ \\
\hline $\mathrm{n} / \mathrm{s}$ & ${ }^{9} \mathrm{Be}(\alpha, \mathrm{n}){ }^{12} \mathrm{C}$ & $\begin{array}{c}\text { Spectrum } \\
{[0.2-11] \mathrm{MeV}}\end{array}$ & 60 \\
\hline \multirow{12}{*}{$\gamma / \mathrm{s}$} & \multirow{3}{*}{${ }^{241} \mathrm{Am} \rightarrow{ }^{237} \mathrm{~Np}^{*}+\alpha$} & $26.3 \mathrm{keV}$ & $2.4 \cdot 10^{4}$ \\
\hline & & $33.2 \mathrm{keV}$ & $1.3 \cdot 10^{3}$ \\
\hline & & $59.5 \mathrm{keV}$ & $3.59 \cdot 10^{5}$ \\
\hline & \multirow{7}{*}{${ }^{237} \mathrm{~Np}^{*} \rightarrow \gamma+{ }^{237} \mathrm{~Np}$} & $125.3 \mathrm{keV}$ & 40.8 \\
\hline & & $146.6 \mathrm{keV}$ & 4.6 \\
\hline & & $208 \mathrm{keV}$ & 7.9 \\
\hline & & $335.4 \mathrm{keV}$ & 5 \\
\hline & & $368.7 \mathrm{keV}$ & 2.2 \\
\hline & & $662.4 \mathrm{keV}$ & 3.64 \\
\hline & & $722.01 \mathrm{keV}$ & 1.96 \\
\hline & \multirow{2}{*}{${ }^{9} \mathrm{Be}(\alpha, \mathrm{n}){ }^{12} \mathrm{C}^{*}$} & $4.438 \mathrm{MeV}$ & 35.46 \\
\hline & & $7.654 \mathrm{MeV}$ & 7.91 \\
\hline
\end{tabular}

energy peaks at $22.3 \mathrm{keV}, 33.3 \mathrm{keV}, 59.54 \mathrm{keV}, 662.4 \mathrm{keV}, 722.01 \mathrm{keV}, 4.438 \mathrm{MeV}$ and $7.654 \mathrm{MeV}$. Although there are other gamma peaks, their probabilities are very low, so they are not taken into account in this analysis. Table 4 shows the relative intensities for these photon peaks $[18,19]$. The source also contained $8 \mathrm{mCi}$ of ${ }^{137} \mathrm{Cs}$ emitting gamma-rays at $662 \mathrm{keV}$ but, as it has been demonstrated in the previous section, this is not expected to contribute to the pulse height distribution measured by the U3DTHIN.

The neutron spectrum of the ${ }^{241} \mathrm{AmBe}$ source covers a range of energies from $0.2 \mathrm{MeV}$ to $11 \mathrm{MeV}$, with a maximum at $4.5 \mathrm{MeV}$. In order to better test the performance of the converter, with higher cross-section in the thermal neutron range, the neutrons from the source were thermalized with a polyethylene block $10 \mathrm{~cm}$ thick placed between the ${ }^{241} \mathrm{AmBe}$ source and the detector. The distance from the center of the source to the silicon detectors was $25 \mathrm{~cm}$. Both an adapted U3DTHIN with neutron converter and a bare U3DTHIN were tested at the same time so the difference in counts between them should be due to the charged particles produced by the capture of neutrons within the converter.

The detectors were biased to $10 \mathrm{~V}$ and the measurement time was 26 hours. Figure 8 shows the count rate distributions obtained by the detectors with an applied threshold of $180 \mathrm{keV}$ to avoid counts from the electronic noise.

The bare U3DTHIN detector obtained $(181 \pm 13) \cdot 10^{-5}$ counts/s. These counts can come from: (i) the natural $\gamma$-ray background, (ii) photons produced from $(n, \gamma)$ reactions in the materials around the devices, (iii) $\gamma$-rays from the neutron capture reaction in the polyethylene block $\mathrm{H}(\mathrm{n}, \gamma) \mathrm{D}$, 


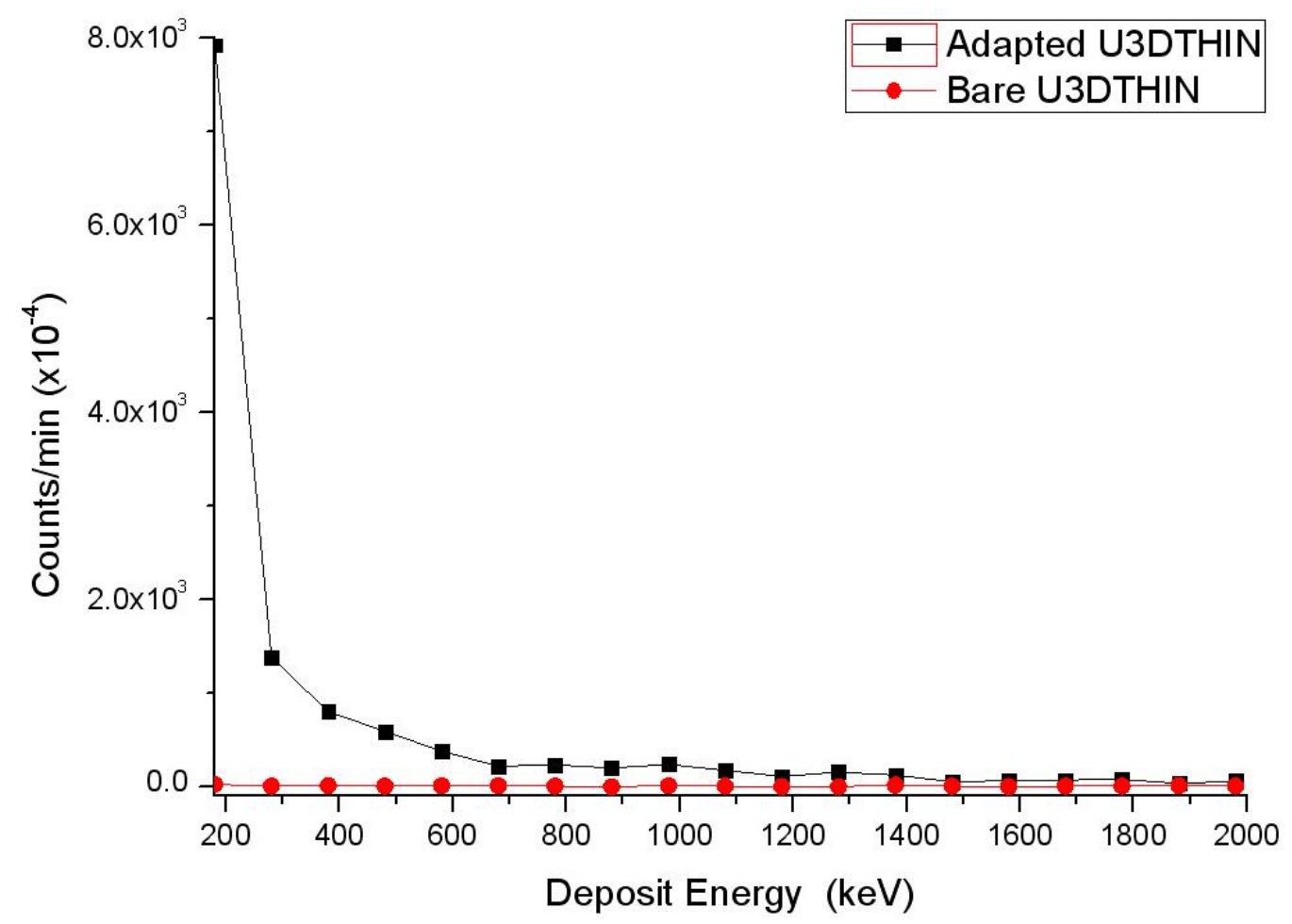

Figure 8. Charged particle pulse height distribution measured by an adapted and a bare U3DTHIN detector. An energy threshold of $180 \mathrm{keV}$ was used in the readout system to avoid electronic noise.

$\mathrm{Q}=2.22 \mathrm{MeV}$, (iv) the 4.438 and $7.654 \mathrm{MeV} \gamma$-rays of the ${ }^{241} \mathrm{AmBe}$ source, with considerable emission rates although low absorption probability in $10 \mu \mathrm{m}$ silicon, (v) energy deposited in the silicon volume by $\mathrm{n}-\mathrm{Si}$ nuclear interactions (only the contributions with energies higher than the $180 \mathrm{keV}$ applied threshold are considered here).

In the same measurement time the o-carborane adapted detector obtained a total of $(218 \pm 5) \cdot 10^{-4}$ counts/s, i.e. this measured one order of magnitude higher than the background measured by the bare detector. This result demonstrates the feasibility of U3DTHIN detector with o-carborane for the detection of slow neutrons. According to the neutron emission rate from the ${ }^{241} \mathrm{AmBe}$ source, $8.8 \cdot 10^{4} \mathrm{n} / \mathrm{s} \cdot 4 \pi \mathrm{sr}$, the percentage of moderated neutrons within the $10 \mathrm{~cm}$ thick polyethylene, and considering the simulated efficiency for thermal neutrons in our device, $2.5 \%$, the expected count rate at $25 \mathrm{~cm}$ is $(280 \pm 80) \cdot 10^{-4}$ counts/s which is in good agreement with the experimental value. The spectral shape of the signal distribution is mainly due to the energy loss for the reaction products from the ${ }^{10} \mathrm{~B}\left(\mathrm{n}\right.$,alpha) ${ }^{7} \mathrm{Li}$ reaction either within the entrance window of the detector or inside the converter.

\section{Conclusions}

An innovative sensor based on an ultra-thin 3D silicon detector with $10 \mu \mathrm{m}$ thickness has been adapted to detect neutrons with a ${ }^{10} \mathrm{~B}$-enriched compound. This adapted U3DTHIN was tested with a ${ }^{137} \mathrm{Cs}$ gamma source, showing a gamma rejection factor for this source higher than $10^{-8}$. 
The sensor was also tested with a ${ }^{241}$ AmBe neutron-gamma source, measuring (199 \pm 5$) \cdot 10^{-4}$ neutrons/s, in agreement with the simulation and analytical calculations. These first experiments show the feasibility for neutron detection with these novel devices and their usefulness in complex mixed gamma-neutron radiation fields.

A new set of improved devices has been fabricated at IMB-CNM's clean room and is currently being tested with encouraging prospects.

\section{Acknowledgments}

Support by the Spanish Ministerio de Ciencia e Innovación and the European Regional Development Fund through project NeutorIII (CIT-300000-2009-011) is acknowledged.

The authors would like to thank Dr. F. Teixidor and Dra. C. Viñas, members of the Institut de Ciència de Materials of Barcelona (ICMAB-CSIC), for providing us with the converter material.

\section{References}

[1] C.F.G. Delaney and E.C. Finch, Radiation Detectors, Physical Principles and Applications, Oxford University Press, New York (1992).

[2] S. Tavernier, Experimental Techniques in Nuclear and Particle Physics, Springer (2010).

[3] A. Syntfeld et al., ${ }^{6} L i I(E u)$ in Neutron and $\gamma$-ray Spectrometry-a Highly Sensitive Thermal Neutron Detector, IEEE Trans. Nucl. Sci. 52 (2005) 3151.

[4] R.T. Kouzes et al., Neutron detection alternatives to 3 He for national security applications, Nucl. Instrum. Meth. A 623 (2010) 1035.

[5] G. Lutz, Semiconductor Radiation Detectors, Springer (1999).

[6] F. García et al., A novel ultra-thin 3D detector-For plasma diagnostics at JET and ITER tokamaks, Nucl. Instrum. Meth. A 607 (2009) 57.

[7] G. Pellegrini et al., Fabrication and simulation of novel ultra-thin 3D silicon detectors, Nucl. Instrum. Meth. A 604 (2009) 115.

[8] F. García, R. Orava, M. Lozano and G. Pellegrini, Back-Thinned radiation detector with 3D active region and corresponding methods of manufacturing and use, Europe Patent PCT/EP2008/066716.

[9] M. Luszik-Bhadra, A prototype personal neutron dosimeter with one silicon diode, Radiat. Protect. Dosim. 96 (2001) 227.

[10] M. Sasaki et al., Development and characterization of real-time personal neutron dosimeter with two silicon detectors, Nucl. Instrum. Meth. A 418 (1998) 465.

[11] T. Lahaye et al., Dosimètre individuel électronique pour les neutrons: Saphydose-n, Radioprotection 35 (2000) 223.

[12] S.I. Parker, C.J. Kenney and J. Segal, 3-D: A New architecture for solid state radiation detectors, Nucl. Instrum. Meth. A 395 (1997) 328.

[13] Instituto de Microelectrónica de Barcelona, IMB-CNM (CSIC), http://www.imb-cnm.csic.es.

[14] C. Guardiola et al., Portable Silicon Neutron Detector System, Proceeding of the 8th Spanish Conference on Electron Devices, CDE'2011, art. no. 5744236. 
[15] C. Guardiola et al, First investigations of a silicon neutron detector with a carborane converter, 2011 JINST 6 P11001.

[16] Monte Carlo N-particle transport code system (MCNP), Alamos National Laboratory, MCNPX 2.6.0, available from http://mcnpx.lanl.gov/.

[17] J.F. Ziegler, SRIM - The Stopping and Range of Ions in Matter, available from http.//www.srim.org/.

[18] Gamma-Ray Spectrum Catalog of Isotopes, available from: http://www.radiochemistry.org/periodictable/gamma_spectra/.

[19] S. Croft, The use of neutron intensity calibrated $B e(\alpha, n)$ sources as $4438 \mathrm{keV}$ Gamma-Ray reference standards, Nucl. Instrum. Meth. A 281 (1989) 103. 